\title{
On the Coordination of Base Stations in Ultra Dense Cellular Networks
}

\author{
Alexis I. Aravanis ${ }^{\dagger}$, Olga Muñoz ${ }^{\dagger}$, Antonio Pascual-Iserte ${ }^{\dagger}$ and Marco Di Renzo* \\ ${ }^{\dagger}$ Dept. of Signal Theory and Communications, \\ Universitat Politècnica de Catalunya (UPC), Barcelona, Spain \\ Email: $\{$ alexios.aravanis, olga.munoz, antonio.pascual $\} @$ upc.edu \\ *Laboratoire des Signaux et Systèmes, \\ CNRS, CentraleSuplec, Univ. Paris-Sud, Universite Paris-Saclay, 91192 Gif-sur-Yvette, France \\ Email: marco.direnzo@12s.centralesupelec.fr
}

\begin{abstract}
Ultra dense networks (UDNs) allow for efficient spatial reuse of the spectrum, giving rise to substantial capacity and power gains. However, recent findings have demonstrated that the reduction of inter-site distances after a certain threshold, has a detrimental effect on the network performance. In such cases, interference mitigation strategies to counteract the effect of primarily the line of sight (LOS) interference are imperative. In this course, the present paper investigates a minimum coordination strategy among immediate neighbors. The adopted scheme realizes the interference mitigation objective, enhancing system capacity, while being implementable in practice. Building upon this scheme, the present paper provides tractable closed form expressions for the downlink (DL) ergodic capacity of a reference user under the examined coordination scheme. The analysis is performed for a scenario consisting of base stations (BSs) whose positions follow a Poisson point process of a given spatial density. The tractability and accuracy of the derived expressions renders them ideal for quantifying the performance of additional coordination strategies and for revealing trends in complex optimization problems. Thus, constituting a valuable tool for network operators, toward assessing different interference mitigation techniques and coordination schemes in UDNs.
\end{abstract}

Index Terms-Ultra dense networks; Base station coordination; Poisson point process; Ergodic Capacity; Rate; Closed form.

\section{INTRODUCTION}

The ever increasing demand for broadband access coupled with the scarcity of frequency resources has imposed the investigation of new approaches to enhance wireless capacity in 5G networks. This can be achieved by leveraging three main pillars of flexibility, i.e. the employment of wider spectrum, the improvement of spectral efficiency and the employment of smaller cell sizes and, thus, of smaller transmit distances. Out of these three pillars, the efficient spatial reuse of the spectrum, achieved through the reduction of the inter-site distances, has provided the most substantial capacity gains by a large margin [1]. Hence, network densification arises as a prime candidate for achieving the envisaged capacity increase, in the $5 \mathrm{G}$ era as well.

In this direction, network operators employ system level simulations and network measurements to plan the densification of their networks. However, following the seminal work of Baccelli et al. [2], which gave rise to stochastic geometry as a tractable tool for the large-scale analysis and design of wireless networks, a multitude of research works have provided significant insights into network densification. These insights are essential for understanding the innate features of dense networks, and can be employed by network operators as practical densification road maps.

Such analytical approaches brought about significant changes in the understanding of wireless networks. In particular, it has been demonstrated that for single-slope path loss models and for networks comprising more users than base stations (BSs), the user signal quality is independent of the BS density [3]. Moreover, the probability of coverage (i.e. the complementary cumulative distribution function (CCDF) of the signal to interference plus noise ratio (SINR)) is independent of the BS density and of the number of tiers [4]. Similar analysis for the uplink (UL) has demonstrated that the UL signal to interference ratio (SIR) is also invariant of the BS density [5]. As a result, it has been demonstrated that the network capacity increases linearly with the density of BSs and with the number of tiers [6].

This analysis however, which indeed holds for sparse wireless networks (e.g. tier of macro cells (MCs)), does not hold for extremely high BS densities. The reason for that is that extremely small inter-site distances allow neighboring BSs to create LOS interference to the intended user. As a result, after a densification threshold, the probability of coverage is diminished precipitately due to the presence of LOS interference [7], [8]. Furthermore, in UDNs where the inter-site distance is comparable to the elevation of the BS and LOS interference is present, the probability of coverage tends to zero for high BS densities [9]. That is since for high BS densities LOS interferers approach the intended user at a faster rate than the rate in which the intended user approaches the elevated BS.

These theoretical findings provide two illuminating conclusions. On the one hand, the network capacity increases linearly with the density of BSs in the presence of only non line of sight (NLOS) interference. On the other hand, the existence of LOS interference sets an upper threshold on network densification. However, the employment of interference mitigation techniques for LOS interference can counteract this detrimental effect, allowing for linear capacity increase with the network density. 
Some early interference mitigation strategies considered guard regions around fixed-size cells, which constituted interference free zones [10]. Similar strategies were also considered for D2D networks [11]. However, the coordination of all BSs residing within these guard regions, which is required by these techniques, is a highly involved task. Moreover, the coordination of all BSs within neighboring guard regions requires the coordination of the entire network as a whole.

As opposed to these techniques, the present paper investigates a minimum coordination strategy. Instead of coordinating a random number of $\mathrm{BSs}$ residing within a fixed zone, a fixed number of BSs are coordinated. The coordinated BSs are the ones residing closest to the selected BS. The number of coordinated BSs is defined a priori, and this value is subsequently introduced in the analytical framework. Contrary to other coordination strategies, the present strategy can be implemented in practice, since in LTE systems BSs are aware of the topology of their immediate neighbors to facilitate the handover process. Moreover, since the immediate neighbors constitute the principal source of LOS interference, such a coordination strategy can provide substantial capacity gains, as buttressed by the aforementioned analysis.

Building upon the benefits of the examined scheme, the present paper derives a tight approximation in closed form, for the user ergodic capacity, for a setup of BSs coordinating at the level of their immediate neighbors. The derived closed form expressions quantify the performance of the network under the examined coordination scheme. Moreover, the tractability of the expressions makes them ideal for revealing trends in complex optimization problems.

The remainder of the paper is organized as follows. Section II presents the considered network architecture and the analytical expressions for the DL ergodic capacity of a reference user equipment (UE). The derived approximations are defined in closed form and provide expressions for the DL ergodic rate in the general case of non-coordinating networks, as well as the particular case of a coordinating network where BSs coordinate with their immediate neighbors. Section III presents the simulation results corroborating that the devised approximations from the preceding analysis are tight. Finally, Section IV concludes the paper and presents perspectives.

\section{The Wireless Cellular Network ArChitecture AND THE ERGODIC CAPACITY APPROXIMATION}

\section{A. The Wireless Cellular Network Scenario}

A wireless cellular system is considered, comprising a set of BSs $B S_{i}$, whose positions follow a spatial distribution given by a homogeneous Poisson point process (PPP) $\Psi$ of density $\lambda\left(B S s / m^{2}\right)$. The reference UE belongs to the cell served by the BS $B S_{0} . B S_{0}$ can be singled out and the location of the other BSs follows the reduced Palm distribution of $\Psi$, which is the same to the original distribution $\Psi$ (Slivnyaks theorem [12]). For the sake of simplicity in the notation, it is assumed that all UEs and BSs are equipped with one antenna. However, the analysis can be easily extended to the multi-antenna case.

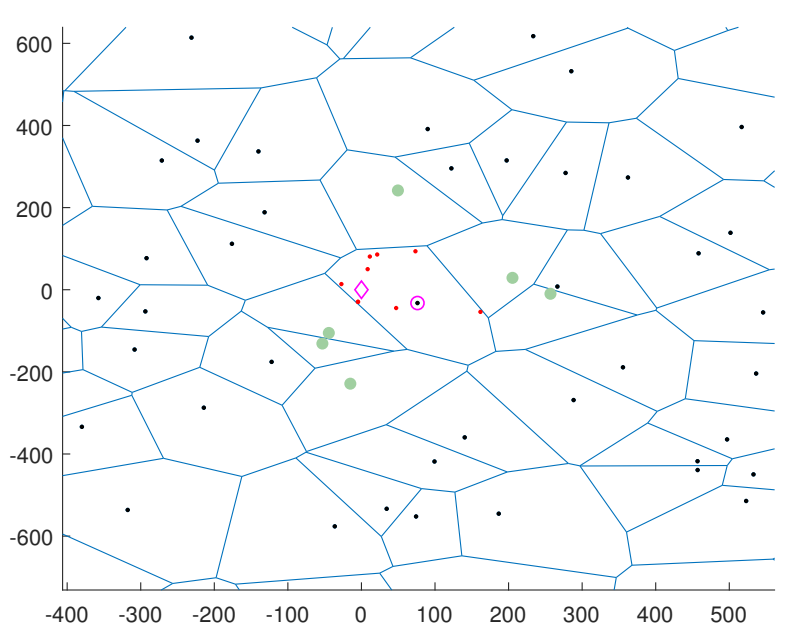

Fig. 1. The Considered Network Scenario

Intra cell users are assumed to be sharing orthogonal resources, as is the typical case in literature [5]. Furthermore, the network is assumed to comprise significantly more users than BSs. As a result, every BS is active and transmitting, acting as an interferer in the DL. However, some BSs adjacent to $B S_{0}$ are assumed to coordinate and not to create any interference. The number of coordinating BSs in the following analysis is defined by an elementary property of stochastic geometry. In particular, the mean degree in the PoissonDelaunay tessellation is 6 [12], hence in the Poisson-Delaunay tessellation of $\Psi$, the average number of adjacent cells to the cell comprising $B S_{0}$ is 6 (similar to hexagonal grids). Hence, the $6 \mathrm{BSs}$ closest to $B S_{0}$ are assumed to coordinate. However, the subsequent analysis can be extended to any number of coordinating BSs.

The considered scenario is depicted in Figure 1, where $B S_{0}$ is marked by the magenta circle, the reference UE by the magenta diamond and the intra cell users are depicted in red. In this setup the interfering BSs are depicted in black and the 6 coordinated BSs in green.

As already stressed, the investigated coordination scheme can be implemented in practice since BSs are aware of the topology of their immediate neighbors to facilitate the handover process. Moreover, such a scheme can counteract effectively the LOS interference which is the most detrimental in UDNs. Hence, LOS interference is assumed to be counteracted in this setup and single-slope path loss models are considered henceforth. For high network densities, where LOS interferers reside also after the 6 closest BSs, the analysis could be extended accordingly.

\section{B. The Ergodic Capacity Bound}

The ergodic capacity of a UE placed at distance $d$ from $B S_{0}$ resulting from the average over fast fading, if no additional 
BSs are overlaid, is given by

$$
\mathbb{E}[R]=\mathbb{E}_{h}\left\{\log \left(1+d^{-\beta} h^{2} \gamma\right)\right\},
$$

where the expectation is with respect to the fading coefficient $h, \log ()$ in all the expressions henceforth represents the natural logarithm, $\beta$ is the path-loss exponent, and $\gamma$ is the SNR at the reference distance with

$$
\gamma=\frac{P_{B S}}{\sigma^{2} L_{r e f}} .
$$

In (2) $P_{B S}$ is the transmission power of the BS, $\sigma^{2}$ is the noise power, and $L_{r e f}$ is the equivalent path-loss at a reference distance of 1 meter, which includes also the effects of the transmit and receive antenna gains.

Assuming that the BSs $B S_{i}$ are placed at distance $d_{i}$ from the UE and every $B S_{i}$ is acting as an interferer (for simplicity in this section's notation, although this is not the case in coming sections due to the coordination of the 6 closest BSs) the expectation of the rate must be calculated with respect to the fading coefficient $h$, the distances $d$ and $d_{i}$ and the fading coefficient $h_{i}$ of all interferers. However, the incorporation of $h_{i}$ in the calculation would imply perfect knowledge of the channel of all interferers, which is not available in practice. Hence, the incorporation of $h_{i}$ would provide an upper bound for the ergodic capacity, whereas its omission would provide a lower bound [11]. Following the approach of [11] the expectation with respect to $h_{i}$ is omitted and the lower bound for the capacity is calculated as follows:

$$
\mathbb{E}[R] \geq \mathbb{E}_{h, d, d_{i}}\left\{\log \left(1+\frac{\gamma h^{2} d^{-\beta}}{\sum_{i=1}^{\infty} \gamma d_{i}^{-\beta}+1}\right)\right\} .
$$

\section{Taylor Approximation}

Following a similar approach as that of the authors in [13], [14], a mathematical manipulation is introduced and the convex function $f(x)=\log (1+\exp (x))$ is employed, revising (3) as follows:

$$
\mathbb{E}[R] \geq \mathbb{E}_{h, d, d_{i}}\left\{f\left(\log \left(\frac{\gamma h^{2} d^{-\beta}}{\sum_{i=1}^{\infty} \gamma d_{i}^{-\beta}+1}\right)\right)\right\} .
$$

The right-hand side of (4) can be approximated by a N-order Taylor expansion, as follows::

$\mathbb{E}\{f(y)\} \approx f(\mathbb{E}\{y\})+\sum_{n=2}^{N} \frac{\mathbb{E}\{y-\mathbb{E}\{y\}\}^{n}}{n !} f^{(n)}(E\{y\})+R_{N}$.

where, $y=\log \left(\frac{\gamma h^{2} d^{-\beta}}{\sum_{i=1}^{\infty} \gamma d_{i}^{-\beta}+1}\right)$ and $R_{N}$ is the error after $N$ terms (Lagrange Remainder) and is given by:

$$
R_{N}=\mathbb{E}\left\{\frac{\left(y-y_{0}\right)^{N+1}}{(N+1) !} f^{(n)}\left(\zeta\left(y, y_{0}\right)\right)\right\}
$$

for some $\zeta\left(y, y_{0}\right) \in\left\langle y, y_{0}\right\rangle$.

The proposed framework allows for a twofold approach. On the one hand an infinitely large number of Taylor terms can be analytically defined, thus, allowing the analytical computation of (4) at the expense of computational time. On the other hand, a tight approximation of (4) can be provided, by employing the first two terms of (5) as follows:

$$
\mathbb{E}\{f(y)\} \approx f(\mathbb{E}\{y\})+\left(\mathbb{E}\{y-\mathbb{E}\{y\}\}^{2}\right) f^{\prime \prime}(E\{y\}) .
$$

Further to the approximation of (7), the employment of Jensen's inequality for the convex function $f(x)$ provides the following lower bound:

$$
\mathbb{E}\{f(y)\} \geq f(\mathbb{E}\{y\}) .
$$

In addition, in the case of a second order expansion there exists an $\alpha \in[0,1]$ such that $[15]$ :

$$
\begin{aligned}
\mathbb{E}\{f(y)\}= & f(\mathbb{E}\{y\}) \\
& +\frac{1}{2}\left(\mathbb{E}\{y-\mathbb{E}\{y\}\}^{2}\right) \mathbb{E}\left\{f^{\prime \prime}(\alpha y+E\{y\}(1-\alpha))\right\} .
\end{aligned}
$$

Hence, employing the Descent Lemma [15] and since the function $f^{\prime \prime}(y)$ attains its maximum value $f^{\prime \prime}(0)=\frac{1}{4}$ at $y=0$ for $\alpha=1$ an upper bound can be defined as follows

$$
\mathbb{E}\{f(y)\} \leq f(\mathbb{E}\{y\})+\frac{1}{8}\left(\mathbb{E}\{y-\mathbb{E}\{y\}\}^{2}\right) .
$$

Having defined the bounds of (8) and (10) that provide a guideline regarding the validity of the obtained expressions, a correction factor $c(\mathbb{E}\{y\})$ is introduced, to fine tune (7). The correction factor $c(\mathbb{E}\{y\})$ is a polynomial function of $\mathbb{E}\{y\}$ that is obtained numerically by the Lagrange remainder of (5) as detailed in the Appendix. Thus, (7) is redefined as follows

$$
\mathbb{E}\{f(y)\} \approx f(\mathbb{E}\{y\})+c(\mathbb{E}\{y\})\left(\mathbb{E}\{y-\mathbb{E}\{y\}\}^{2}\right) f^{\prime \prime}(E\{y\}) .
$$

In order to employ (7)-(11) for the computation of (4) $\mathbb{E}\{y\}$ needs to be defined. Since the expectations over the fading and the distances are independent $\mathbb{E}\{y\}$ is obtained as follows

$$
\begin{aligned}
\mathbb{E}\{y\}= & \mathbb{E}_{h, d, d_{i}}\left\{\log \left(\frac{\gamma h^{2} d^{-\beta}}{\sum_{i=1}^{\infty} \gamma d_{i}^{-\beta}+1}\right)\right\} \\
= & \log (\gamma)+\mathbb{E}_{h}\{2 \log (h)\}-\mathbb{E}_{d}\{\beta \log (d)\} \\
& -\mathbb{E}_{d_{i}}\left\{\log \left(\sum_{i=1}^{\infty} \gamma d_{i}^{-\beta}+1\right)\right\} .
\end{aligned}
$$

Assuming a Rayleigh fading where $h$ follows a zeromean circularly symmetric Gaussian distribution with variance equal to 1 , then the pdf of the fast fading is given by $f_{H}(h)=2 h e^{-h^{2}}$. Moreover, the pdf of the distance $d$ between a reference user and its closest BS is given by [16]

$$
f_{D}(d)=2 \pi d \lambda e^{-\lambda \pi d^{2}}, d \geq 0
$$

Since the pdf of a Rayleigh distribution is given by:

$$
f_{Y}(y)=\frac{y}{\sigma_{y}^{2}} e^{\frac{-y^{2}}{2 \sigma_{y}^{2}}},
$$

both random variables $h$ and $d$ follow a Rayleigh distribution with $\sigma_{d}^{2}=\frac{1}{2 \pi \lambda}$ and $\sigma_{h}^{2}=\frac{1}{2}$. Hence, the random variables of the second and third term of (13) follow a log-Rayleigh distribution. 
The mean of a $\log$-Rayleigh distributed variable $\mu$ is given by [17]

$$
\mathbb{E}\{\mu\}=\log \sigma+\frac{\log 2}{2}-\frac{\psi}{2}
$$

where $\psi$ is the Euler-Mascheroni constant defined as $\psi=$ $-\int_{0}^{\infty} \log (x) \exp (-x) d x \approx 0.577$. The variance of the $\log$ Rayleigh distributed variable $\mu$ is given by [17]

$$
\mathbb{E}\{\mu-\mathbb{E}\{\mu\}\}^{2}=\frac{\pi^{2}}{24}
$$

Since $\log d$ and $\log h$ are $\log$-Rayleigh distributed random variables of variance $\sigma_{d}^{2}$ and $\sigma_{h}^{2}$, by employing (13) and (16) it follows that

$$
\begin{array}{r}
\mathbb{E}\{y\}=\log (\gamma)+\frac{\beta}{2}(\psi+\log (\pi \lambda))-\psi \\
-\mathbb{E}_{d_{i}}\left\{\log \left(\sum_{i=1}^{\infty} \gamma d_{i}^{-\beta}+1\right)\right\}
\end{array}
$$

and by (13) and (17):

$$
\begin{aligned}
\mathbb{E}\{y-\mathbb{E}\{y\}\}^{2}= & \left(\beta^{2}+4\right) \frac{\pi^{2}}{24} \\
& -\operatorname{var}\left\{\log \left(\sum_{i=1}^{\infty} \gamma d_{i}^{-\beta}+1\right)\right\}
\end{aligned}
$$

In order to compute (18) and (19) the expectation and the variance of the log of the aggregate interference needs to be computed. In this course, it is known that the expectation and the variance of the aggregate interference can be computed in an exact manner by Campbell's theorem as follows [18]:

$$
\begin{aligned}
\mathbb{E}_{d_{i}}\left\{\sum_{i \in \Psi} d_{i}^{-\beta}\right\} & =\lambda \int_{\mathbb{R}^{+}} d_{i}^{-\beta} 2 \pi d_{i} d d_{i}, \\
\operatorname{var}_{d_{i}}\left\{\sum_{\mathrm{i} \in \Psi} \mathrm{d}_{\mathrm{i}}^{-\beta}\right\} & =\lambda \int_{\mathbb{R}^{+}}\left(d_{i}^{-\beta}\right)^{2} 2 \pi d_{i} d d_{i},
\end{aligned}
$$

Employing the results of (20) and (21), the expectation and variance of the log of the aggregate interference can be approximated by the Taylor expansion of (5) as follows:

$$
\begin{aligned}
& \mathbb{E}_{d_{i}}\left\{\log \left(\sum_{i=1}^{\infty} \gamma d_{i}^{-\beta}+1\right)\right\} \approx \\
& \log \left(\mathbb{E}_{d_{i}}\left\{\sum_{i=1}^{\infty} \gamma d_{i}^{-\beta}\right\}+1\right)-\frac{\operatorname{var}_{d_{i}}\left\{\sum_{\mathrm{i}=1}^{\infty} \gamma d_{i}^{-\beta}\right\}}{2\left(\mathbb{E}_{d_{i}}\left\{\sum_{i=1}^{\infty} \gamma d_{i}^{-\beta}\right\}+1\right)^{2}}
\end{aligned}
$$

and

$$
\operatorname{var}_{d_{i}}\left\{\log \left(\sum_{\mathrm{i}=1}^{\infty} \gamma \mathrm{d}_{\mathrm{i}}^{-\beta}+1\right)\right\} \approx \frac{\operatorname{var}_{d_{i}}\left\{\sum_{\mathrm{i}=1}^{\infty} \gamma d_{i}^{-\beta}\right\}}{\left(\mathbb{E}_{\mathrm{d}_{\mathrm{i}}}\left\{\sum_{\mathrm{i}=1}^{\infty} \gamma \mathrm{d}_{\mathrm{i}}^{-\beta}\right\}+1\right)^{2}}
$$

By plugging (20) and (21) into (22) and (23), the expectation and variance of (18) and (19) are defined.

\section{The Correction Factor $c(\mathbb{E}\{y\})$}

For the Log-Rayleigh random variables of (18) an infinitely large number of moments can be computed analytically [19]. In addition, the moment generating function (MGF) of the mean interference appearing in (18) is also known [6]. Hence, after obtaining the cumulants from the respective moments an infinitely large number of Taylor terms of (5) can be computed analytically for the exact calculation of the bound of (4).

However, in the present analysis, the employment of the function $f(y)$ allows for the introduction of a correction factor

\begin{tabular}{|c|c|}
\hline$\beta$ & $c(\mathbb{E}\{y\})$ \\
\hline 3 & $\begin{array}{l}-0.0001(\mathbb{E}\{y\})^{5}+0.0006(\mathbb{E}\{y\})^{4}-0.0044(\mathbb{E}\{y\})^{3} \\
+0.0918(\mathbb{E}\{y\})^{2}-0.0199(\mathbb{E}\{y\})+0.6730\end{array}$ \\
\hline 4 & $\begin{array}{l}-0.0002(\mathbb{E}\{y\})^{5}+0.0016(\mathbb{E}\{y\})^{4}-0.0052(\mathbb{E}\{y\})^{3} \\
+0.0937(\mathbb{E}\{y\})^{2}-0.0254(\mathbb{E}\{y\})+0.6059\end{array}$ \\
\hline 5 & $\begin{array}{l}-0.0004(\mathbb{E}\{y\})^{5}+0.0023(\mathbb{E}\{y\})^{4}-0.0036(\mathbb{E}\{y\})^{3} \\
\quad+0.0926(\mathbb{E}\{y\})^{2}-0.0293(\mathbb{E}\{y\})+0.5465\end{array}$ \\
\hline
\end{tabular}
$c(\mathbb{E}\{y\})$ into the first two terms of the Taylor expansion. This correction by a polynomial function provides extremely accurate results. The correction factor $c(\mathbb{E}\{y\})$ needs to be computed numerically for different values of the path-loss exponent. This computation is performed offline for different path-loss exponents and the obtained results are tabulated in Table I. The derivation of $c(\mathbb{E}\{y\})$ is presented in the Appendix.

TABLE I

THE CORRECTION FACTOR $c(\mathbb{E}\{y\})$

\section{E. Coordination of Immediate Neighbors}

For the particular scenario considered herein, where the first interfering $\mathrm{BSs}$ is in fact the 7 th closest $\mathrm{BS}$ to $B S_{0}$ the first interferer is not in the vicinity of the UE. As a result of this large value of $d_{i},(23)$ and the last term of (22) tend to zero and (18) and (19) are defined as follows:

$$
\begin{aligned}
\mathbb{E}\{y\}= & \log (\gamma)+\frac{\beta}{2}(\psi+\log (\pi \lambda))-\psi \\
& -\log \left(\lambda \int_{\mathbb{R}^{+}} \gamma d_{i}^{-\beta} 2 \pi d_{i} d d_{i}+1\right),
\end{aligned}
$$

and by (13) and (17):

$$
\mathbb{E}\{y-\mathbb{E}\{y\}\}^{2}=\left(\beta^{2}+4\right) \frac{\pi^{2}}{24} .
$$

However, the integration limits of the last term of (24) needs to be defined accordingly, taking into account only the interference from BSs farther than the 6th closest BSs to $B S_{0}$. By assuming that the $\mathrm{UE}$ and the $B S_{0}$ are relatively close compared to the distance to the first interferer (which for the considered scenario is actually the case), it can be assumed that the 6th closest BS to $B S_{0}$ is in fact the 7th closest BS to the $\mathrm{UE}$ (the closest $\mathrm{BS}$ to the $\mathrm{UE}$ is $B S_{0}$ ). Hence, in order to define the distance between the UE and the first interferer, the distance between the UE and the 7th BS must be defined. 
In this direction, is employed the pdf of the distance to the $\mathrm{n}$-th neighbor in a 2-dimensional PPP, which is given by [20]

$$
f_{R_{n}}(r)=\exp \left(-\pi \lambda r^{2}\right) \frac{2\left(\lambda \pi r^{2}\right)^{n}}{r \Gamma(n)}
$$

Hence, the expected distance between the UE and its 7th closest BS is given by

$$
\mathbb{E}\left\{d_{7}\right\}=\int_{0}^{\infty} d \exp \left(-\pi \lambda d^{2}\right) \frac{2\left(\lambda \pi d^{2}\right)^{7}}{\Gamma(7) d} d d=\frac{3003}{2048 \sqrt{\lambda}}
$$

The result of (27) can be employed for the lower integration limit of the last term of (24). Moreover, since $B S_{0}$ coordinates with its 6 closest BSs this implies that the whole network also coordinates in a similar fashion. Hence, out of the BSs acting as interferers only one out of seven BSs transmits at any given resource block. Thus, the density of the interferers is in fact $\lambda_{I}=\frac{\lambda}{7}$ and the last term of (24) is given by

$$
\begin{aligned}
\mathbb{E}\left\{\sum_{i \in \Psi} d_{i}^{-\beta} \mathbf{1}\left\{d_{i} \geq \mathbb{E}\left\{d_{7}\right\}\right\}\right\}= \\
\quad \int_{\frac{3003}{2048 \sqrt{\lambda}}}^{\infty} d^{-\beta+1} \lambda_{I} 2 \pi d r \stackrel{\beta \geq 2}{=} \frac{2 \pi \lambda}{7(\beta-2)}\left(\frac{2048 \sqrt{\lambda}}{3003}\right)^{\beta-2} .
\end{aligned}
$$

Hence, by plugging (28) into (24) the following holds:

$$
\begin{aligned}
\mathbb{E}\{y\}= & \log (\gamma)+\frac{\beta}{2}(\psi+\log (\pi \lambda))-\psi \\
& -\log \left(\frac{2 \gamma \pi \lambda}{7(\beta-2)}\left(\frac{2048 \sqrt{\lambda}}{3003}\right)^{\beta-2}+1\right) .
\end{aligned}
$$

The expectation of (29) along with the variance of (25) can be employed for the computation of the DL ergodic rate by the approximations and bounds of (7)-(11). For the sake of completeness in the expressions the approximation of (7) is given by

$$
\begin{aligned}
\mathbb{E}\{R\} \approx & \log (1+\exp (E\{y\})) \\
& +\left(\mathbb{E}\{y-\mathbb{E}\{y\}\}^{2}\right) \frac{\exp (E\{y\})}{(1+\exp (E\{y\}))^{2}} .
\end{aligned}
$$

\section{Simulations}

In order to demonstrate the tight performance of the extremely tractable approximation of (30), and of all bounds and approximations defined in (7)-(11), the wireless network of Section II-A has been simulated, encompassing BSs positioned according to a homogeneous PPP of spatial density $\lambda$ and a reference UE. The 6 closest BSs to $B S_{0}$ which serves the UE do not produce any interference. As opposed to those BSs, one in every seven of the remaining BSs is selected randomly and acts as an interferer in the DL. The parameters employed for the calculation of the link budget are tabulated in Table II.

The tight relationship between the obtained analytical expression of (7)-(11) and the simulated results is manifested in Figure 2, where the ergodic DL rate of the reference UE is plotted for different BS densities and for two different path loss exponents. The tight performance of the expressions verifies the reliability of the preceding analysis and its utility

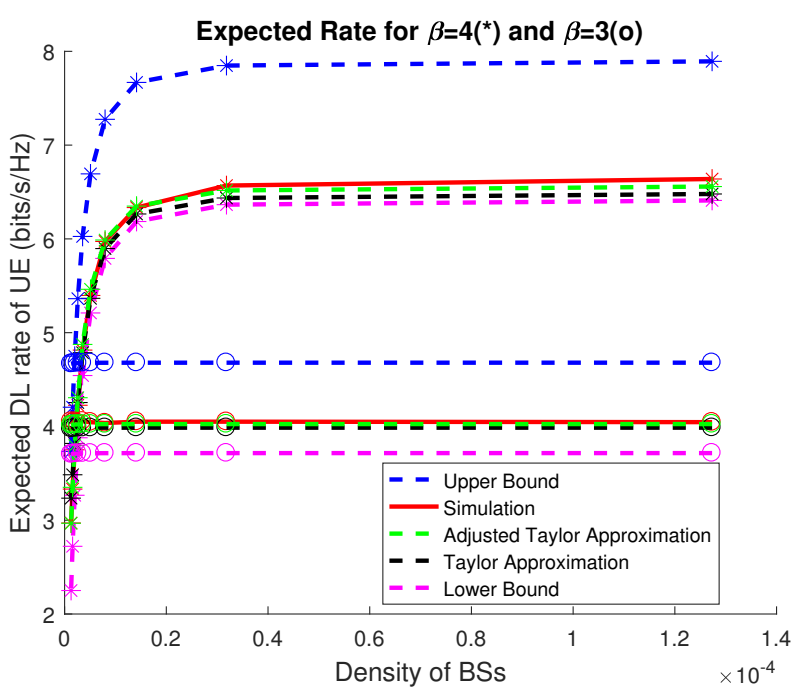

Fig. 2. Expected DL rate vs density of BSs for different path loss exponents $\beta=4$ and $\beta=3$

toward devising efficient coordination strategies. Furthermore, the performance of the network as the BS density increases corroborates the fundamental conclusion of stochastic geometry that the user signal quality is independent of the BS density. Thus, further verifying the validity of the analysis.

TABLE II

LINK BUDGET PARAMETERS

\begin{tabular}{|c|c|}
\hline Parameter & Value \\
\hline \hline BS Transmit Power $P_{B S}$ & $33 \mathrm{dBm}$ \\
\hline Bandwidth & $10 \mathrm{MHz}$ \\
\hline Noise Power Spectral Density & $-174 \mathrm{dBm} / \mathrm{Hz}$ \\
\hline Noise Power $\sigma^{2}$ & $-104 \mathrm{dBm}$ \\
\hline $\begin{array}{c}\text { Path Loss at Reference Distance } L_{r e f} \\
\text { (Including Antenna Gains) }\end{array}$ & $25.6 \mathrm{~dB}$ \\
\hline Path Loss Exponent & $3 \& 4$ \\
\hline
\end{tabular}

\section{Conclusions}

The increasing interest toward network densification and the emerging analytical framework have demonstrated the potential of UDNs with respect to capacity enhancement and power efficiency. However, it has become evident that in order reap those benefits, efficient interference mitigation schemes are essential. In this direction, the present paper analyzed a minimum coordination strategy, providing extremely accurate and tractable expressions for quantifying the DL ergodic capacity of a reference user, thus, characterizing the performance of the investigated coordination scheme.

The tractability and accuracy of the proposed analytical framework renders it ideal for characterizing additional coordination strategies, thus, allowing for assessing and developing efficient coordination strategies based on their performance. Last but not least, its tractability allows to be used complementary to intractable stochastic geometry approaches, 
revealing trends and allowing for the convergence of complex optimization problems.

\section{APPENDIX}

\section{Langrange Remainder}

Following the definition of (5) and (6) the funcion $f(x)=$ $\log (1+\exp (x))$ can be expanded around $x_{0}$ as follows:

$$
f(x)=f\left(x_{0}\right)+f^{\prime}\left(x_{0}\right)\left(x-x_{0}\right)+R_{1}\left(x, x_{0}\right)
$$

where

$$
R_{1}\left(x, x_{0}\right)=\frac{\left(x-x_{0}\right)^{2}}{2} f^{\prime \prime}\left(\zeta\left(x, x_{0}\right)\right)
$$

is the Langrange remainder for some $\zeta\left(x, x_{0}\right) \in\left\langle x, x_{0}\right\rangle$.

Note, that the function $f^{\prime \prime}(x)=\frac{e^{x}}{\left(1+e^{x}\right)^{2}}$ tends to zero for values outside the interval $(-6,6)$. Therefore, the remainder needs to be defined only within this interval. By employing (31) the $\mathbb{E}\left\{R_{1}\left(x, x_{0}\right)\right\}$ can be computed numerically in this particular interval. That is, by assuming that $x$ follows a $\log$-Rayleigh distribution, which is the case if the interference is disregarded. The analysis can be repeated taking also the statistics of the interference into account. However, in this case the accuracy gain is minimal and by far outweighed by the complexity introduced in the analysis.

\section{Adjusting Correction Factor $c(\mathbb{E}\{y\})$}

In order to compute $\mathbb{E}\{f(\cdot)\}=\mathbb{E}\{\log (1+\exp (\cdot))\}$, the series is expanded around $x_{0}=\mathbb{E}\{y\}$, and by employing (31), (11) and (25) we obtain:

$$
\begin{aligned}
\mathbb{E}\{f(y)\} & =f(E\{y\})+\mathbb{E}\left\{R_{1}(x, E\{y\})\right\} \\
& =f(E\{y\})+c(E\{y\}) f^{\prime \prime}(E\{y\})\left(\beta^{2}+4\right) \frac{\pi^{2}}{24}
\end{aligned}
$$

Having defined $\mathbb{E}\left\{R_{1}\left(x, x_{0}\right)\right\}$ numerically, $c(\mathbb{E}\{y\})$ can be defined employing (33) for different values of $\beta$. The numerically obtained correction factor $c(\mathbb{E}\{y\})$ is then approximated by a polynomial approximation and the results are tabulated in Table I for different values of $\beta$.

\section{ACKNOWLEDGMENT}

The work presented in the present paper has been carried out within the framework of the project ETN5 Gwireless (this project has received funding from the European Union's Horizon 2020 research and innovation programme under the Marie Skłodowska-Curie grant agreement No. 641985). Moreover, the work has been partially funded through the grant 2017 SGR 578 (funded by the Catalan Government-Secretaria d'Universitats i Recerca, Departament d'Empresa i Coneixement, Generalitat de Catalunya, AGAUR) and the project TEC2016-77148-C2-1R (AEI/FEDER, UE): 5G\&B-RUNNER-UPC (funded by the Agencia Estatal de Investigacion, AEI, and Fondo Europeo de Desarrollo Regional, FEDER).

\section{REFERENCES}

[1] V. Chandrasekhar, J. G. Andrews, and A. Gatherer, "Femtocell networks: a survey," IEEE Communications Magazine, vol. 46, no. 9, pp. 59-67, September 2008.

[2] F. Baccelli, B. Blaszczyszyn, and P. Muhlethaler, "An Aloha protocol for multihop mobile wireless networks," IEEE Transactions on Information Theory, vol. 52, pp. 421-436, February 2006.

[3] J. G. Andrews, F. Baccelli, and R. K. Ganti, "A tractable approach to coverage and rate in cellular networks," IEEE Transactions on Communications, vol. 59, no. 11, pp. 3122-3134, November 2011.

[4] H. S. Dhillon, R. K. Ganti, F. Baccelli, and J. G. Andrews, "Modeling and analysis of k-tier downlink heterogeneous cellular networks," IEEE Journal on Selected Areas in Communications, vol. 30, no. 3, pp. 550560, April 2012.

[5] S. Singh, X. Zhang, and J. G. Andrews, "Joint rate and SINR coverage analysis for decoupled uplink-downlink biased cell associations in HetNets," IEEE Transactions on Wireless Communications, vol. 14, no. 10, pp. 5360-5373, Oct. 2015.

[6] M. Haenggi, Stochastic Geometry for Wireless Networks. Cambridge University Press, 2013.

[7] M. D. Renzo, W. Lu, and P. Guan, "The intensity matching approach: A tractable stochastic geometry approximation to system-level analysis of cellular networks," IEEE Transactions on Wireless Communications, vol. 15 , no. 9, pp. 5963-5983, Sept 2016.

[8] M. Ding, P. Wang, D. Lopez-Perez, G. Mao, and Z. Lin, "Performance impact of los and nlos transmissions in dense cellular networks," IEEE Transactions on Wireless Communications, vol. 15, no. 3, pp. 23652380, March 2016.

[9] M. Ding, D. Lopez-Perez, G. Mao, and Z. Lin, "Performance impact of idle mode capability on dense small cell networks," IEEE Transactions on Vehicular Technology, vol. 66, no. 11, pp. 10 446-10 460, Nov 2017.

[10] R. W. Heath, M. Kountouris, and T. Bai, "Modeling heterogeneous network interference using poisson point processes," IEEE Transactions on Signal Processing, vol. 61, no. 16, pp. 4114-4126, Aug 2013.

[11] G. Geordie, "Device-to-device communication and wearable networks: Harnessing spatial proximity," Ph.D. dissertation, 2017.

[12] S. N. Chiu, D. Stoyan, W. Kendall, and J. Mecke, Stochastic Geometry and its Applications. John Wiley and Sons, 2013.

[13] A. I. Aravanis, O. Munoz, A. Pascual-Iserte, and J. Vidal, "Analysis of downlink and uplink decoupling in dense cellular networks," in 2016 IEEE 21st International Workshop on Computer Aided Modelling and Design of Communication Links and Networks (CAMAD), Oct 2016, pp. 219-224.

[14] A. Aravanis, A. Pascual-Iserte, and O. Munoz-Medina, "Closed-form capacity bounds for downlink and uplink decoupling," in WSA 2018; 22nd International ITG Workshop on Smart Antennas, March 2018, pp. $1-5$.

[15] D. P. Bertsekas, Nonlinear Programming, 2nd ed. Athena Scientific, 1999.

[16] S. Sadr and R. S. Adve, "Partially-distributed resource allocation in small-cell networks," IEEE Transactions on Wireless Communications, vol. 13, no. 12, pp. 6851-6862, Dec. 2014.

[17] B. Rivet, L. Girin, and C. Jutten, "Log-rayleigh distribution: A simple and efficient statistical representation of log-spectral coefficients," IEEE Transactions on Audio, Speech, and Language Processing, vol. 15, no. 3, pp. 796-802, March 2007.

[18] M. Haenggi, J. G. Andrews, F. Baccelli, O. Dousse, and M. Franceschetti, "Stochastic geometry and random graphs for the analysis and design of wireless networks," IEEE Journal on Selected Areas in Communications, vol. 27, no. 7, pp. 1029-1046, September 2009.

[19] W. L. Shepherd and P. Milnarich, "Moments of log rayleigh distributions," Proceedings of the IEEE, vol. 62, no. 8, pp. 1168-1169, Aug 1974.

[20] M. Haenggi, "On distances in uniformly random networks," IEEE Transactions on Information Theory, vol. 51, no. 10, pp. 3584-3586, Oct 2005. 